\title{
FRET-Based Detection of Enzymatic Reaction of Botulinum on Microfluidic Device
}

\author{
Young Min Bae, Seung Oh Jin, Insoo Kim, and Ki Young Shin \\ Korea Electrotechnology Research Institute, 111 Hanggaulro, Ansan 426-910, Republic of Korea \\ Correspondence should be addressed to Young Min Bae; kimbym@keri.re.kr
}

Received 22 April 2015; Revised 3 July 2015; Accepted 16 July 2015

Academic Editor: Zhida Xu

Copyright (C) 2015 Young Min Bae et al. This is an open access article distributed under the Creative Commons Attribution License, which permits unrestricted use, distribution, and reproduction in any medium, provided the original work is properly cited.

\begin{abstract}
A microfluidic device was implemented to detect the enzymatic reaction of botulinum toxin A (BTA) using Förster resonance energy transfer (FRET). The microfluidic device comprised a main channel having two loading zones, a reaction chamber and a side channel perpendicular to the main channel. The reaction chamber defined by weir in the main channel was packed with microbeads. The movement of the peptide substrate and the BTA in the microfluidic device was controlled by electrophoresis, and the enzymatic reaction of the BTA was detected through the changes of the fluorescence intensity in the reaction chamber. As a result, it was observed that the enzymatic reaction was affected by the electric voltage applied for the movement of the BTA and the peptide and improved by packing the microbeads in the reaction chamber. The microfluidic device provides the tool to investigate the proteolysis of the substrate by the BTA.
\end{abstract}

\section{Introduction}

Microfluidics technology that deals with the behavior, precise control, and manipulation of tiny volume of fluids in microliter scale has been used to execute various biological protocols to detect specific biomaterials [1]. By scaling down conventional biological protocols executed in laboratories into chip-based analyses, the microfluidics provides advantages as follows: (1) a tiny sample volume is required, (2) an analysis time is reduced, (3) experimental procedures are automated, and (4) high-throughput system (HTS) can be implemented [2,3]. For example, the antigen-antibody binding for immunoassay and the catalytic reaction of enzymes were executed in microfluidic devices $[4,5]$.

Botulism, which is a rare but serious paralytic illness, is caused by botulinum toxins (BTs) that are produced by a bacterium, Clostridium botulinum (C. botulinum) [6]. There are three main kinds of botulism, of which food-borne botulism is caused by eating foods that contain the botulinum toxin. As C. botulinum can survive the boiling temperature of water at sea level, it is hard to remove its toxicity. The toxicity of BTs is due to their enzymatic function. The BTs, of which there are seven serologically distinct types, are composed of two peptide sequences-a heavy chain and a light chain [7]. The heavy chain is particularly important for targeting the toxin to specific types of axon terminals, and the light chain is the proteolytic enzyme that cleaves the specific peptide sequence of neuronal proteins inside the axon terminal, which blocks the releases of acetylcholine in a neuronal system [8]. As the BTs, of which the median lethal dose LD50 is around $1-10 \mathrm{ng} / \mathrm{kg}$, are fatal to humans, they are considered as a candidate for biological weapons [9]. Indeed, the methods for detecting the enzymatic reaction of BTs have been implemented using the technique such as Förster resonance energy transfer (FRET) $[10,11]$.

In this study, we proposed the structure of microfluidic device to implement the FRET reaction and demonstrated the feasibility of it to detect the enzymatic reaction of botulinum toxin A (BTA). It is unique that the device is designed to execute the enzymatic reaction of the BTA and detect a fluorescence signal due to the FRET technique. The detailed scheme of the FRET technique based on the enzymatic reaction is represented by Figure 1. In the "before reaction" state, there is the peptide substrate that can be specifically cleaved by BT. Fluorescein isothiocyanate (FITC) as a donor and 4,4dimethylaminoazobenzene- $4^{\prime}$-carboxylic acid (DABCYL) as an acceptor are chemically attached in both ends of peptide substrate, respectively. In the "before reaction" state, when 

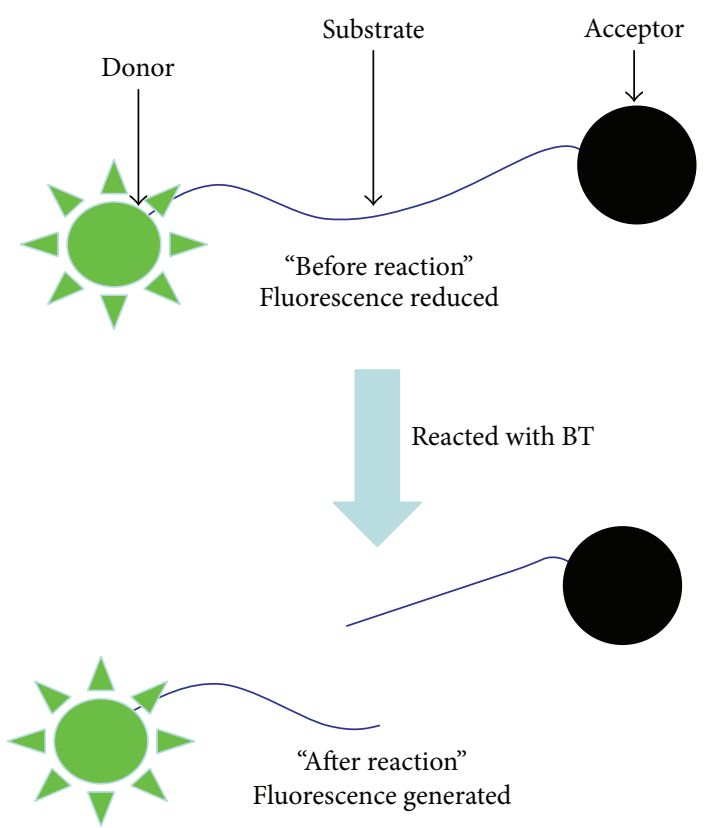

FIGURE 1: FRET-based detection of the proteolytic reaction of the BTA using FRET.

the light with specific wavelength for exciting the donor is irradiated, the emitted photon is absorbed by the acceptor, which results in reducing fluorescence of the donor. However, after the specific sequence of peptide is cleaved by BTs (the "after reaction" state in Figure 1), the photon due to the emission of donor is not absorbed by the acceptor. Here, the enzymatic reaction of the BTA was executed in the microfluidic device fabricated with PDMS (polydimethylsiloxane), and the fluorescence due to the emission from the donor was observed using a fluorescence microscope. Finally, the applicability of the microfluidic device to detect the enzymatic reaction of BTA was investigated.

\section{Materials and Methods}

2.1. Materials. PDMS prepolymer (Sylgard 184) was purchased from Dow Corning company, and polystyrene microbeads having a nominal diameter of $9.77 \mu \mathrm{m}$ were purchased from Bangs Laboratories.

A light chain of BTA and a substrate having a peptide sequence cleaved by the light chain of BTA were purchased from List Biological Laboratories, Inc. The peptide sequence of substrate is TRDIDQANQRTTK ( $\mathrm{T}$, threonine; $\mathrm{R}$, arginine; I, isoleucine; $\mathrm{D}$, aspartic acid; $\mathrm{A}$, alanine; $\mathrm{N}$, asparagine; Q, glutamine; and K, lysine) [12]. The substrate has FITC as a donor and DABCYL as an acceptor bound chemically in both ends of it. All other chemicals were purchased from SigmaAldrich company.

All aqueous solution was prepared using $18 \mathrm{M} \Omega-\mathrm{cm}$ water. A $0.01 \mathrm{M}$ phosphate-buffered saline (PBS, $\mathrm{pH} 7.4$ ) solution containing $138 \mathrm{mM} \mathrm{NaCl}, 2.7 \mathrm{mM} \mathrm{KCl}, 8.1 \mathrm{mM}$ $\mathrm{Na}_{2} \mathrm{HPO}_{4}, 1.47 \mathrm{mM} \mathrm{KH}_{2} \mathrm{PO}_{4}$, and $0.05 \%$ Tween 20 was used in all experiments.
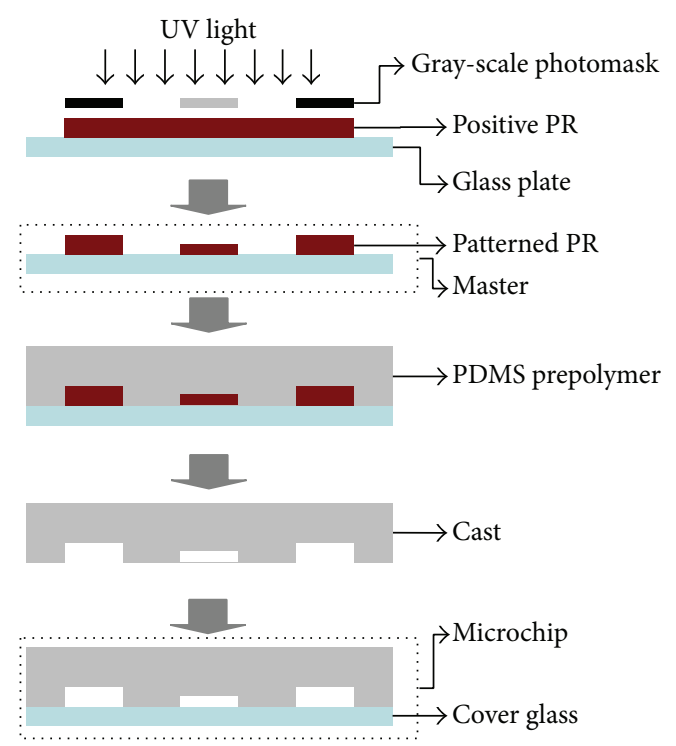

FIGURE 2: Fabrication of microfluidic device using photolithography and PDMS molding technique.

2.2. Fabrication of Microfluidic Device. The microfluidic device was fabricated using the PDMS molding method described elsewhere (Figure 2) [13, 14]. The positive photoresistive (PR) film (AZ P4620, Clariant Co.) deposited on a glass plate was patterned by gray-scale photolithography [15]. A photographic film (Kodak Precision Line Film LPD4) was used as a gray-scale photomask for the photolithographic process. In order to prepare the photomask, after the microchannel pattern was drawn using the commercial software (CorelDraw 9, Corel Corporation, USA), it was printed onto a high-quality, glossy paper with a dye-sublimation printer. Then, the printed design was photoreduced by a factor of 10 onto the photographic film. The glass plate with the PR film patterned was used as the master.

To fabricate the microfluidic device, a 10:1 mixture of the PDMS prepolymer and the curing agent was poured onto the master and then cured at $60^{\circ} \mathrm{C}$ for at least 2 hours. After being separated from the master, the cast (the cured PDMS) and a clean microscopy glass were treated with an oxygen plasma (60 W, model PDC-32G, Harrick Scientific, Ossining, NY) for $30 \mathrm{sec}$. After that, immediately, the device was completed by irreversibly sealing the cast with the microscopy glass. After being sealed, the microchannel was filled with deionized water to keep the inner surface hydrophilic and stored until needed.

2.3. Experimental Setup. Fluorescence change due to the enzymatic reaction of BT was observed using a fluorescence microscope (Nikon Eclipse TE 300, Nikon Co., Tokyo, Japan) equipped with band-pass filters, a $100 \mathrm{~W}$ mercury lamp, and a CCD camera. As the FITC as the donor has the excitation in $488 \mathrm{~nm}$ wavelength and the emission in $523 \mathrm{~nm}$ wavelength, the corresponding filter set was used in the setup of fluorescence microscope. 


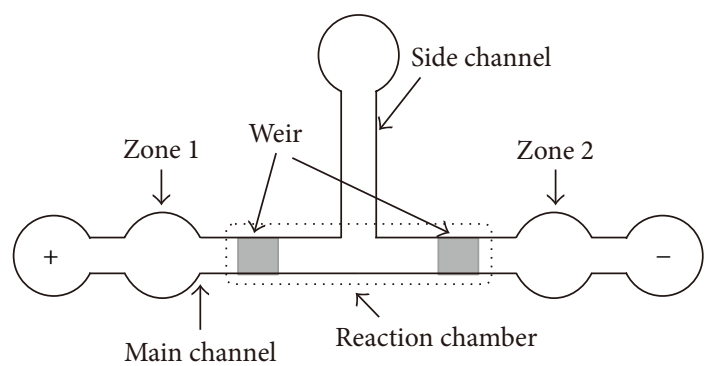

(a)

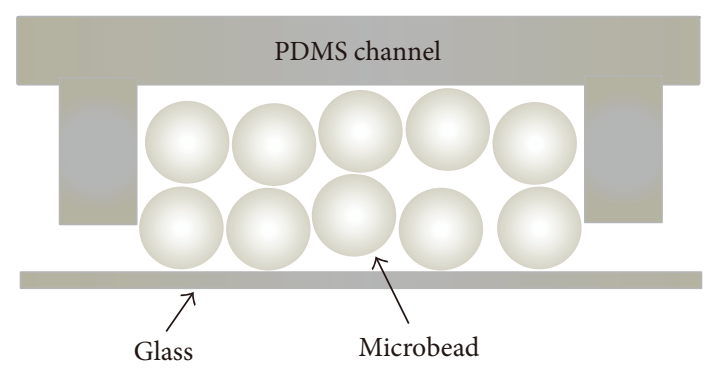

(b)

Figure 3: Microfluidic device designed for the enzymatic reaction of BT: (a) the layout of microfluidic device (not scaled), (b) a scheme of reaction chamber packed with microbeads (not scaled).

\section{Results and Discussion}

3.1. Operation of Microfluidic Device. The layout of microfluidic device designed for executing the enzymatic reaction of the BTA was shown in Figure 3(a). The microfluidic device of " $\mathrm{T}$ " shape comprises a main channel having two loading zones and a reaction chamber and a side channel perpendicular to it. The length of main channel is $14 \mathrm{~mm}$, and the width of channels is $150 \mu \mathrm{m}$. Zones 1 and 2 for loading the substrate and the BTA are, respectively, located on both ends of the main channel as shown in Figure 3(a). In addition, two weirs are fabricated in between both loading zones, and the distance between the weirs is $2 \mathrm{~mm}$. A reaction chamber is defined as the part of the main channel between both weirs (Figure 3(b)). The reaction chamber was packed with polystyrene microbeads. The microbeads injected into the microchannel through the side channel are moved into the main channel, and the weirs of the height lower than the diameter of microbeads block the movements of microbeads into the ends of main channel, so the microbeads were packed in the reaction chamber. To prepare the weir in the microchannel, the weir should be thinner than the other area in the microchannel pattern on the master. Conventionally, the type of master with multiple thicknesses is fabricated using a two-step photolithographic process: microchannel patterns are fabricated in the first step, and only parts of weir are exposed and developed in the second step. However, the two-step process is complicated and needs a precise alignment to rightly allocate weirs on microchannel. Here, the process for fabricating the master having the weir structure was simplified by employing gray-scale photolithography (Figure 2). In the gray-scale photolithography, as the intensity of UV light passing through a gray-scale pattern is reduced, the photochemical reaction is controlled on a local area of PR layer [15]. Therefore, the thickness of PR layer exposed to UV light through the gray-scale pattern can be controlled in the developing process. Based on the preliminary experiment on the change of thickness of positive PR layer as a function of a gray level of photomask, the gray level of the weir area was determined to be $50 \%$ (the gray levels of 0 and $100 \%$ are full transparency and no transparency, resp.). So, the microchannel pattern with the transparencies of $50 \%$ only in the weir part and $100 \%$ in the other area was used to prepare the gray-scale photomask.
Figures 4(a) and 4(b) show the micrograph of the part of weir in the device fabricated by the PDMS molding technique. The height of microchannels and reaction chamber except for the weir was $21.3 \pm 1 \mu \mathrm{m}$, which is the same as the thickness of PR layer. The height and length in the part of weir were $5.7 \pm 0.3 \mu \mathrm{m}$ and $150 \mu \mathrm{m}$, respectively. Some roughness was observed on the channel surface of the part of weir. This was due to the fact that as the gray-level of photomask was controlled with the density of black microdot, the PR layer was not evenly removed in the developing process. Figures $4(\mathrm{c})$ and $4(\mathrm{~d})$ show the micrographs of the right and left sides of reaction chamber packed with microbeads. The microbead solution was injected with a syringe through the side channel. During the microbeads injection, they were not observed to pass through the weir.

The operation of the microfluidic device is as follows: (1) the substrate and the BTA are loaded in zones 1 and 2 with micropipettes, respectively, (2) electric potential is applied to both ends of the main channel with the electric direction as shown in Figure 3(a), and (3) a change of fluorescence intensity in the reaction chamber due to the enzymatic reaction of the BTA is observed. The isoelectric point (pI) of the peptide substrate is 9.0 and the pI value of the BTA is 6 [16]. Therefore, under the buffer solution of $\mathrm{pH} 7.4$, the substrate and the toxin are positively and negatively charged, respectively. When the electric field is applied between both ends of the main channel, the substrate moves toward the electric negative side and the BTA moves toward the electric positive side. Both materials are mixed in the reaction chamber packed with the microbeads, followed by the enzymatic reaction of the BTA. Each part of substrates cleaved by the reaction is moved toward the direction determined according to their pI value. The portion of peptide attached to FITC, of which the pI value is 4 , moves toward the electric positive side. Therefore, in our experiment, the changes of fluorescence in the end of the reaction chamber close to the electric positive side were observed.

Figures 5(a) and 5(b) show the fluorescence image in the reaction experiment in which the BTA was loaded in zone 2 and in the control experiment in which the BTA was not loaded, respectively. In the reaction experiment, $10 \mu \mathrm{L}$ of substrate $(20 \mu \mathrm{M})$ and $10 \mu \mathrm{L}$ of the BTA $(2 \mu \mathrm{g} / \mathrm{mL})$ were, respectively, loaded in zone 1 and zone 2 , followed by 


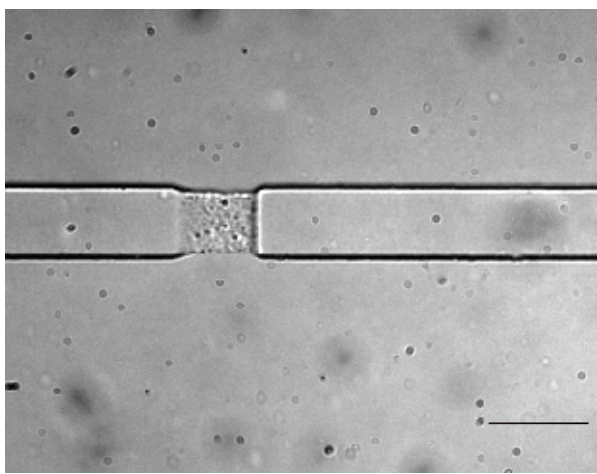

(a)

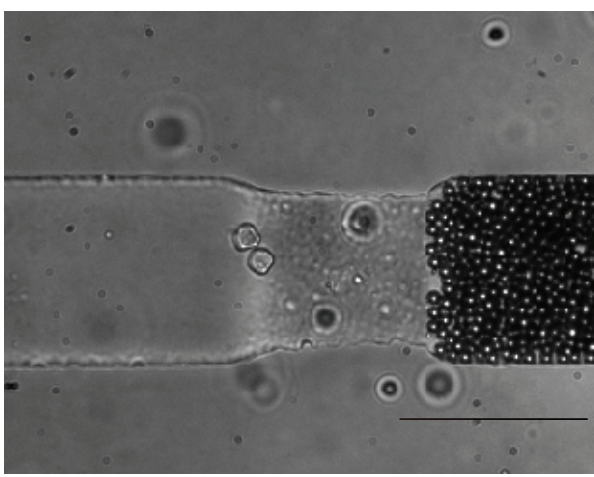

(c)

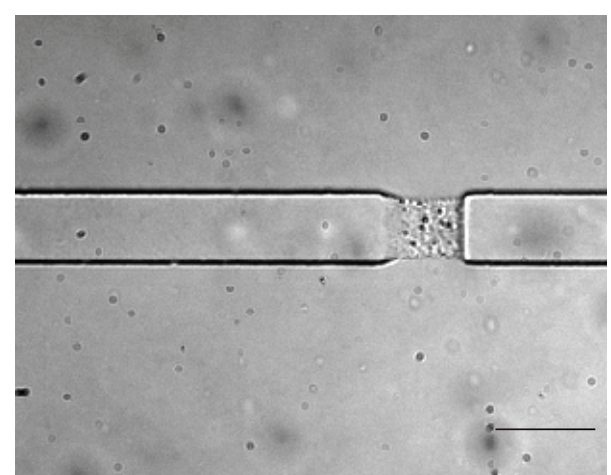

(b)

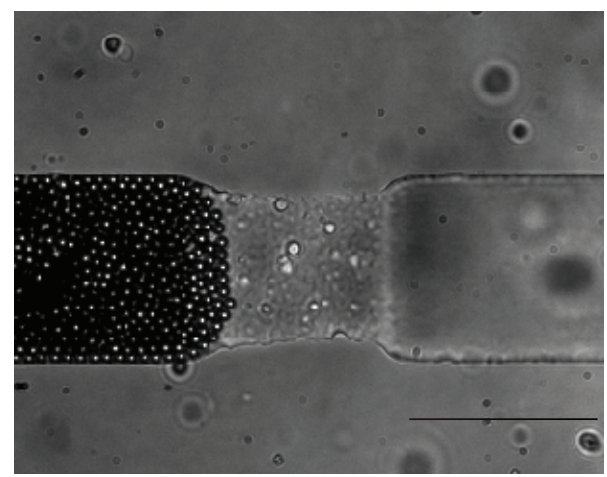

(d)

FIGURE 4: Micrograph of the weirs fabricated in the microfluidic device: (a) and (b) show the weir fabricated in the right and left sides of main channel; (c) and (d) show both ends of reaction chamber packed with microbeads. The scale bar in the figures means $100 \mu \mathrm{m}$.

applying the electric potential of $10 \mathrm{~V}$ to both ends of the main channel as the direction shown in Figure 3(a). In the reaction experiment (Figure 5(a)), the fluorescence intensity in the rectangle area marked with the dotted line became constant in around $5 \mathrm{~min}$. Figure 5(c) shows the profile of fluorescence intensity in the rectangle area marked with the dotted line in Figures 5(a) and 5(b). In both profile curves, the fluorescence intensities due to FITC label attached in the substrate increased in the area of microchannel; however, it was observed that the fluorescence intensity in the reaction experiment was higher than that in the control experiment. The difference of fluorescence meant that the enzymatic reaction was generated in the reaction chamber and the part of substrate with FITC moved toward the electric positive side.

Therefore, the microfluidic device could be applied to detect the enzymatic reaction of $\mathrm{BT}$, and the enzymatic reaction is estimated as the difference between both fluorescence intensities in the reaction and in control experiments.

3.2. Characteristics of Microfluidic Device. The effect of the magnitude of electric field applied to both ends of the main channel was investigated. Figure 6(a) shows the change of fluorescence intensity as a function of the voltage applied. The fluorescence intensity was observed in around $5 \mathrm{~min}$ after the voltage was applied to both sides of the main channel. The enzymatic reaction in the electric field of $5 \mathrm{~V}$ was highest and the fluorescence intensity decreased with the increasing voltage. In movement of biomaterials charged electrically using electrophoresis, the velocity of charged materials is proportional to the strength of electric field applied with the following equation [16]:

$$
v=E \times \mu_{e},
$$

where $\mu_{e}=$ electrophoretic mobility of particle moved, $v=$ the velocity, and $E=$ electric field strength.

In the experiment, as the substrate and the BTA are, respectively, charged positively and negatively in the buffer solution of $\mathrm{pH} 7.4$, they are transported into the reaction chamber. However, with the increasing voltage, the substrate and the BTA moved too fast to complete the reactions in the reaction chamber, which resulted in the decrease of the enzymatic reaction.

In addition, the effect of microbeads packed in the reaction chamber was investigated. Figure 6(b) shows the average fluorescence intensities of microchannel in both experiments in which the microbeads were packed in the reaction chamber and were not. The electric potential of $5 \mathrm{~V}$ was applied in both experiments. The fluorescence intensities in both experiments were higher than that in the control experiment, which meant that the enzymatic reaction was generated in both experiments. However, the fluorescence intensity in the reaction chamber packed with microbeads was higher than that in the reaction chamber without 


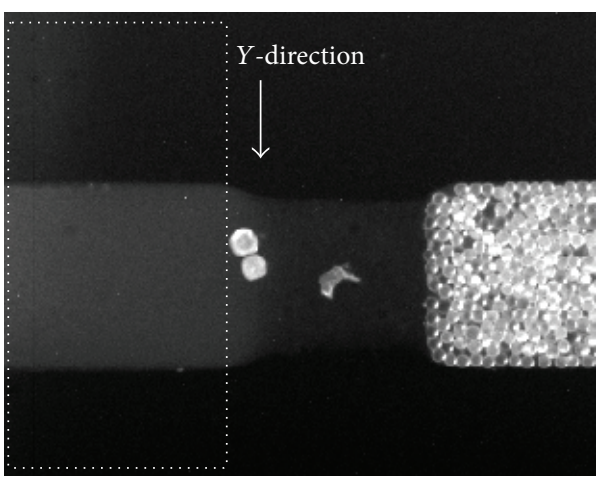

(a)

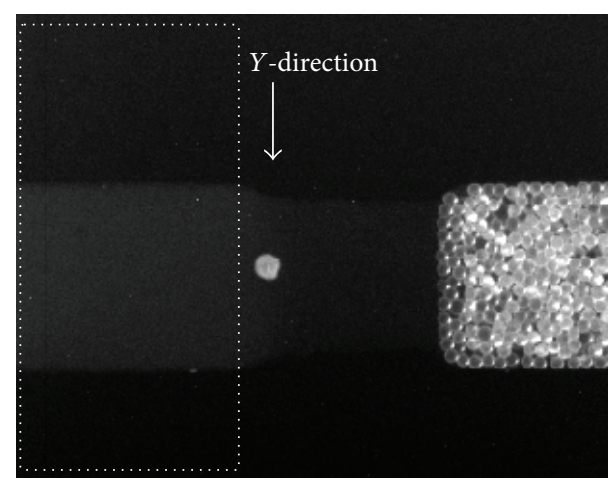

(b)

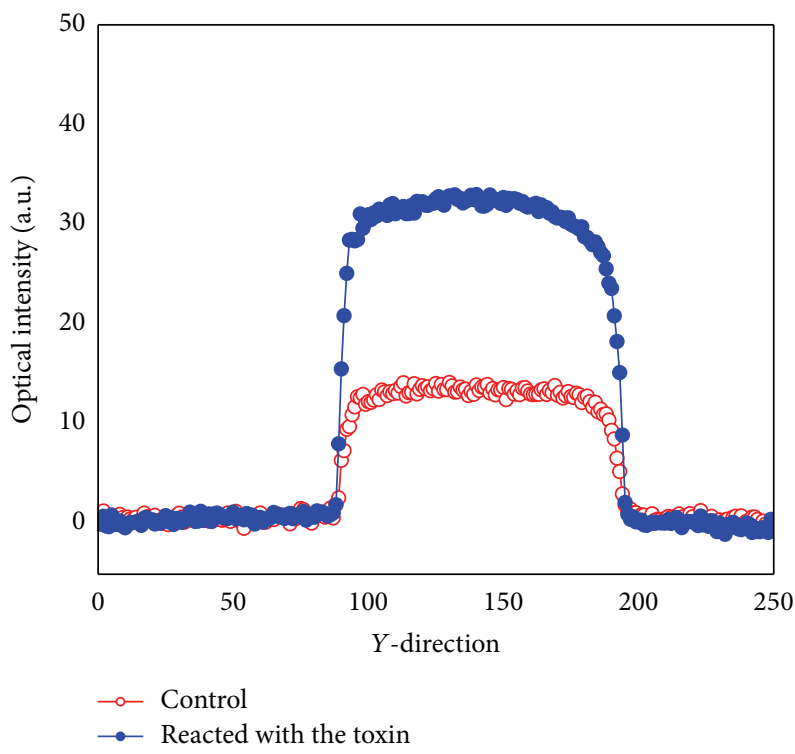

(c)

FIGURE 5: Operation of microfluidic device to execute the enzymatic reaction of the BTA according to $10 \mathrm{~V}$ application. (a) The fluorescence image of microchannel in the reaction experiment with loading the BTA, (b) the fluorescence image of microchannel in the control experiment without loading the BTA, and (c) the comparison of profiles of fluorescence intensity of both the experiments.

microbeads. Generally, because the Reynolds number of flow is small in microchannel, the turbulent flow is difficult to occur. So, mixing in microchannel is dependent on diffusion of mixtures. For efficient mixing in the microchannel, the surface area for mixing and the diffusion time should increase $[17,18]$. In the microfluidic device designed, microbeads packed in the reaction chamber not only increased the surface area for mixing, but also extended the physical path of the substrate and the BTA to pass through, which increased the diffusion time. In other reports, microbeads bed was used for efficiently mixing two laminar flows and increasing enzyme reaction in microchannel [19]. Conclusively, the reaction chamber packed with microbeads made the substrate and the BTA mixed well, which increased the BTA's enzymatic reaction.

\section{Conclusions}

Botulinum toxins (BTs) are a kind of protease that cleaves a specific sequence of peptide (substrate) by hydrolysis. Here, a microfluidic device was designed to detect the proteolysis by BT using the Förster resonance energy transfer (FRET) and implemented using the PDMS molding technique. A master for the device having multiple thicknesses of pattern was prepared using gray-scale photolithography. The microfluidic device of " $\mathrm{T}$ " shape comprises a main channel with a reaction chamber and a side channel, and the reaction chamber packed with polystyrene microbeads was defined with two weirs fabricated in the main channel. The electrophoresis was used to transport the substrate and the BTA into the reaction chamber. Fluorescence signals due to the enzymatic reaction were observed on the microfluidic device, and the enzymatic reaction was affected by the magnitude of voltage applied. In addition, it was observed that the microbeads packed in the reaction chamber could improve the enzymatic reaction of BT by increasing the diffusion time and surface area for mixing both materials. As a result, the microfluidic device developed in this study can be applied to detect the enzymatic reaction of $\mathrm{BT}$. 


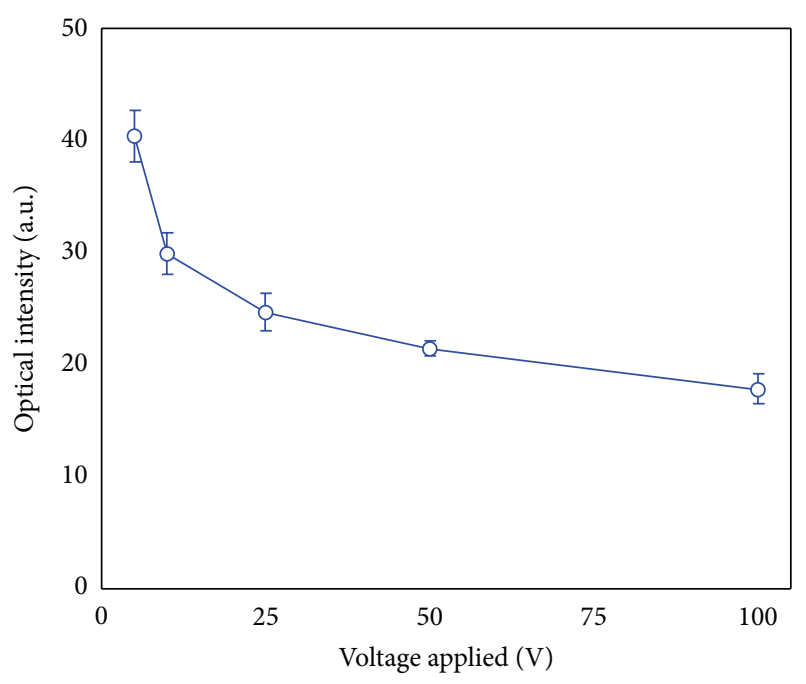

(a)

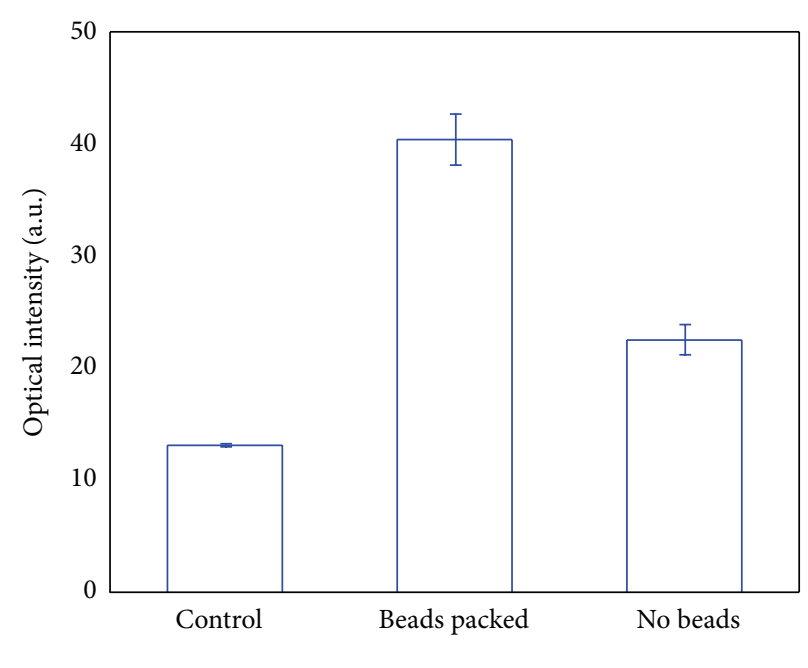

(b)

Figure 6: Characteristics of the microfluidic device. (a) Effect of electric field applied on the enzymatic reaction of the BTA. (b) Effect of microbeads packed in the reaction chamber as a passive mixer.

\section{Conflict of Interests}

The authors declare that there is no conflict of interests regarding the publication of this paper.

\section{Acknowledgment}

This research was supported by Korea Electrotechnology Research Institute (KERI) Primary Research Program through the National Research Council of Science \& Technology (NST) funded by the Ministry of Science, ICT and Future Planning (MSIP) (no. 15-12-N0101-29).

\section{References}

[1] R. Bashir, "BioMEMS: state-of-the-art in detection, opportunities and prospects," Advanced Drug Delivery Reviews, vol. 56, no. 11, pp. 1565-1586, 2004.

[2] A. Bernard, B. Michel, and E. Delamarche, "Micromosaic immunoassays," Analytical Chemistry, vol. 73, no. 1, pp. 8-12, 2001.

[3] G. H. Seong, W. Zhan, and R. M. Crooks, "Fabrication of microchambers defined by photopolymerized hydrogels and weirs within microfluidic systems: application to DNA hybridization," Analytical Chemistry, vol. 74, no. 14, pp. 33723377, 2002.

[4] K. Sato, M. Yamanaka, H. Takahashi, M. Tokeshi, H. Kimura, and T. Kitamori, "Microchip-based immunoassay system with branching multichannels for simultaneous determination of interferon-gamma," Electrophoresis, vol. 23, no. 5, pp. 734-739, 2002.

[5] J. Heo and R. M. Crooks, "Microfluidic biosensor based on an array of hydrogel-entrapped enzymes," Analytical Chemistry, vol. 77, no. 21, pp. 6843-6851, 2005.

[6] CDC, "Botulism," 1999, http://www.cdc.gov.
[7] R. Jin, A. Rummel, T. Binz, and A. T. Brunger, "Botulinum neurotoxin $B$ recognizes its protein receptor with high affinity and specificity," Nature, vol. 444, no. 7122, pp. 1092-1095, 2006.

[8] G. E. Boldt, L. M. Eubanks, and K. D. Janda, "Identification of a botulinum neurotoxin A protease inhibitor displaying efficacy in a cellular model," Chemical Communications, vol. 2006, no. 29, pp. 3063-3065, 2006.

[9] S. S. Arnon, R. Schechter, T. V. Inglesby et al., "Botulinum toxin as a biological weapon: medical and public health management," The Journal of the American Medical Association, vol. 285, no. 8, pp. 1059-1070, 2001.

[10] M. Pires-Alves, M. Ho, K. K. Aberle, K. D. Janda, and B. A. Wilson, "Tandem fluorescent proteins as enhanced FRET-based substrates for botulinum neurotoxin activity," Toxicon, vol. 53, no. 4, pp. 392-399, 2009.

[11] M. Dongo, W. H. Tepp, E. A. Johnson, and E. R. Chapman, "Using fluorescent sensors to detect botulinum neurotoxin activity in vitro and in living cells," Proceedings of the National Academy of Sciences of the United States of America, vol. 101, no. 41, pp. 14701-14706, 2004.

[12] N. R. Shine, K. R. Crawford, and L. J. A. Eaton, "Substrate peptides and assays for detecting and measuring proteolytic activity of serotype a neurotoxin from clostridium botulinum," U.S. Patent No. 6504006, 2003.

[13] R. F. Service, "Materials science: throwing-or molding-a curve into nanofabrication," Science, vol. 273 , no. 5273, p. 312 , 1996.

[14] D. C. Duffy, J. C. McDonald, O. J. A. Schueller, and G. M. Whitesides, "Rapid prototyping of microfluidic systems in poly(dimethylsiloxane)," Analytical Chemistry, vol. 70, no. 23, pp. 4974-4984, 1998.

[15] M.-H. Wu, C. Park, and G. M. Whitesides, "Fabrication of arrays of microlenses with controlled profiles using gray-scale microlens projection photolithography," Langmuir, vol. 18, no. 24, pp. 9312-9318, 2002.

[16] S. S. Dukhin and B. V. Derjaguin, Electrokinetic Phenomena, John Wiley \& Sons, 1974. 
[17] D. R. Meldrum and M. R. Holl, "Microscale bioanalytical systems," Science, vol. 297, no. 5584, pp. 1197-1198, 2002.

[18] M. K. Jeon, J.-H. Kim, J. Noh, S. H. Kim, H. G. Park, and S. I. Woo, "Design and characterization of a passive recycle micromixer," Journal of Micromechanics and Microengineering, vol. 15, no. 2, pp. 346-350, 2005.

[19] G. H. Seong and R. M. Crooks, "Efficient mixing and reactions within microfluidic channels using microbead-supported catalysts," Journal of the American Chemical Society, vol. 124, no. 45, pp. 13360-13361, 2002. 

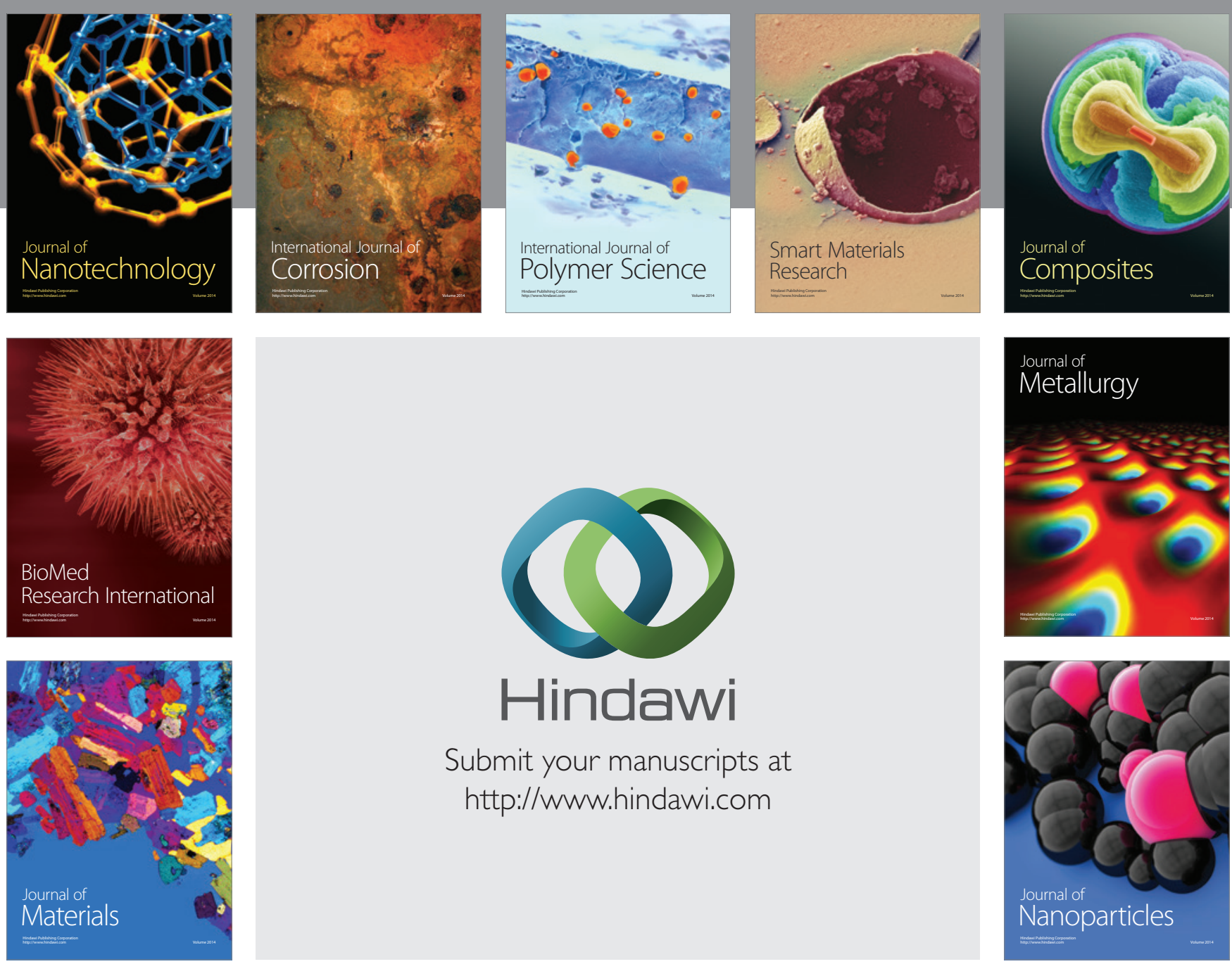

Submit your manuscripts at http://www.hindawi.com
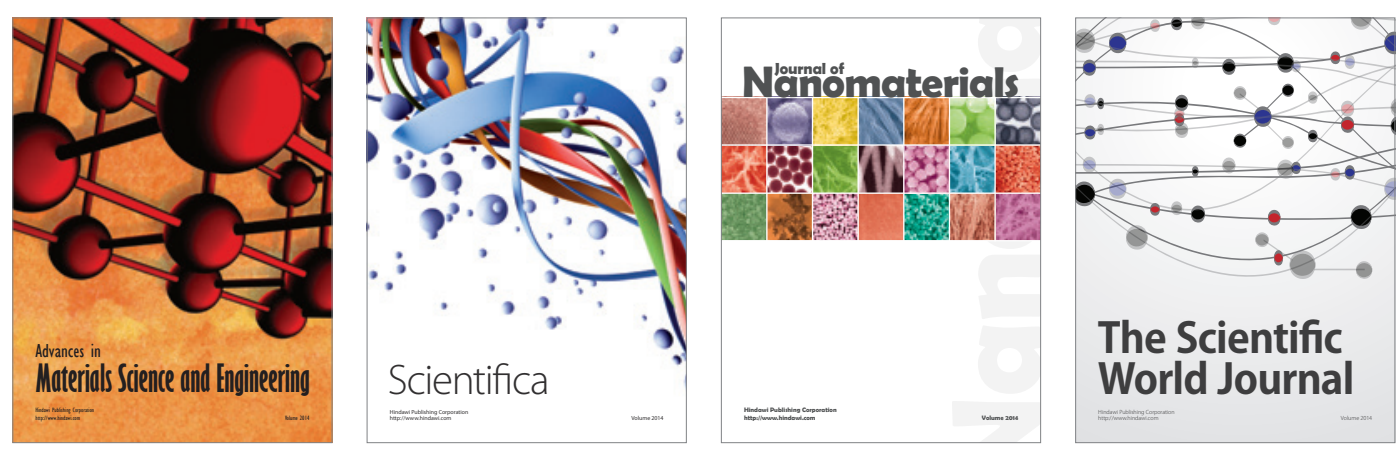

\section{The Scientific World Journal}
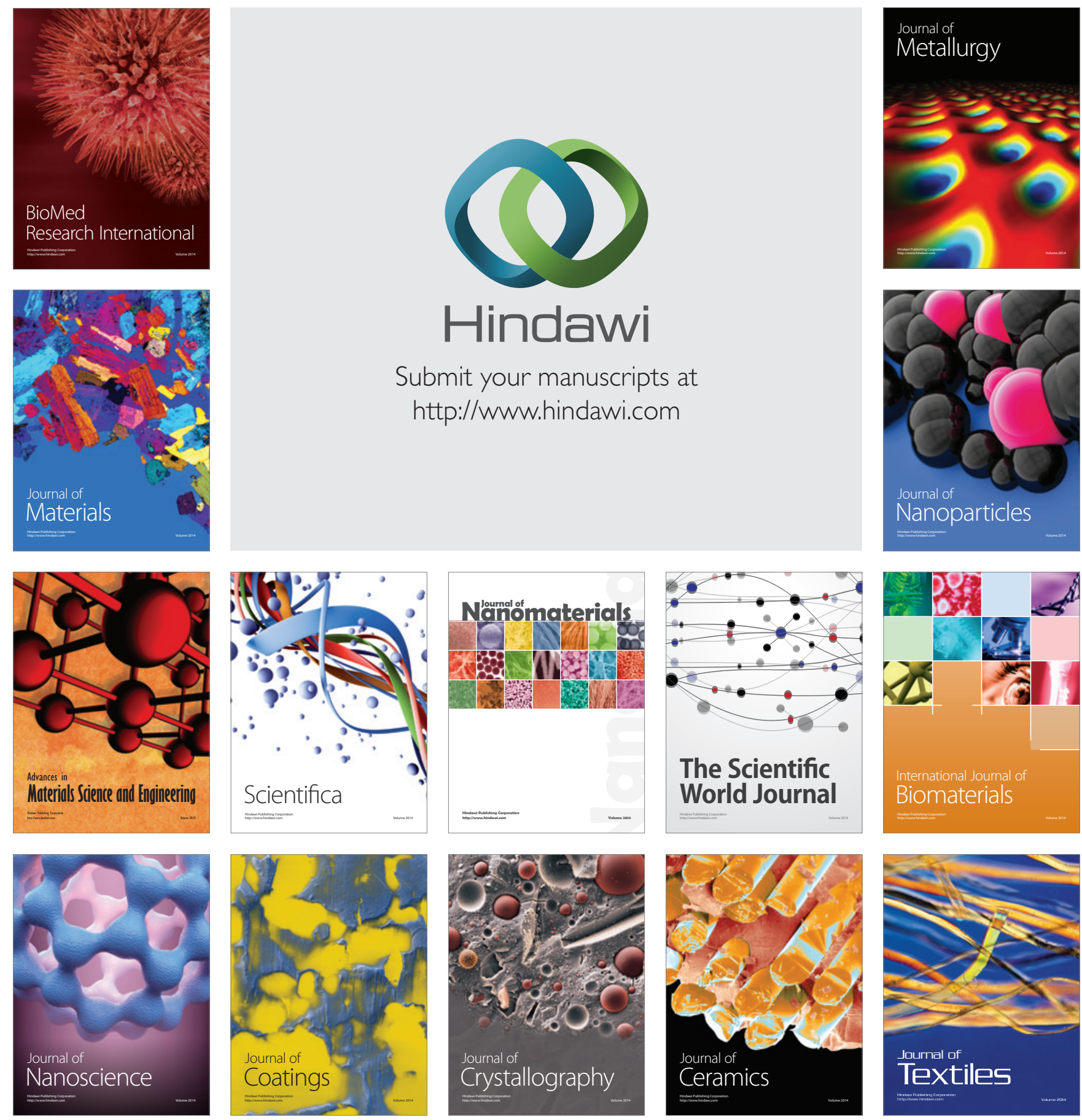